\title{
Self-Training Algorithm Based on Density Peaks Combining Globally Adaptive Multi-Local Noise Filter
}

\section{Shuaijun Li}

Chongqing Normal University

Jia Lu ( $\nabla$ jia-lun@163.com)

Chongqing Normal University

\section{Research Article}

Keywords: Self-training algorithm, Density peaks clustering, Noise filter.

Posted Date: November 22nd, 2021

DOI: https://doi.org/10.21203/rs.3.rs-1048816/v1

License: (c) (1) This work is licensed under a Creative Commons Attribution 4.0 International License.

Read Full License 


\title{
Self-training algorithm based on density peaks combining globally adaptive multi-local noise filter
}

\author{
Shuaijun Li , Jia Lu* \\ Corresponding author: Jia Lu (jia-lun@163.com)` \\ College of Computer and Information Sciences, Chongqing Normal University, \\ Chongqing 401331, China
}

\begin{abstract}
Self-training algorithm can quickly train an supervised classifier through a few labeled samples and lots of unlabeled samples. However, self-training algorithm is often affected by mislabeled samples, and local noise filter is proposed to detect the mislabeled samples. Nevertheless, current local noise filters have the problems: (a) Current local noise filters ignore the spatial distribution of the nearest neighbors in different classes. (b) They can't perform well when mislabeled samples are located in the overlapping areas of different classes. To solve the above challenges, a new self-training algorithm based on density peaks combining globally adaptive multi-local noise filter (STDP-GAMNF) is proposed. Firstly, the spatial structure of data set is revealed by density peak clustering, and it is used for helping self-training to label unlabeled samples. In the meantime, after each epoch of labeling, GAMLNF can comprehensively judge whether a sample is a mislabeled sample from multiple classes or not, and will reduce the influence of edge samples effectively. The corresponding experimental results conducted on eighteen real-world data sets demonstrate that GAMLNF is not sensitive to the value of the neighbor parameter $k$, and it can be adaptive to find the appropriate number of neighbors of each class.
\end{abstract}

Keywords: Self-training algorithm, Density peaks clustering, Noise filter.

\section{Introduction}

Supervised learning is a popular research in machine learning and data mining. Although supervised learning can effectively make a lot of labeled samples to build a classifier, labeling samples usually need a lot of time and consume plenty of money. On the contrary, semi-supervised learning (SSL) (Li Y et al. 2019; Yuan Cet al. 2020) can train a classifier using a few number of labeled samples and a large number of unlabeled samples, which has been successfully applied to many real applications, such as text classification(Tran et al. 2017), face recognition(Liu et al. 2021), medicine(Zhang et al. 2020) and other fields.

SSL algorithm includes self-training algorithm(Li et al. 2019), deep generative models(Pande et al. 2021), co-training algorithm (Slivka et al. 2017) and graph-based semi-supervised learning(Anis et al.

\section{Shuaijun $\mathrm{Li}$}

1209868866@qq.com

Jia Lu (Corresponding author)

jia-lun@163.com

This work is supported by the scientific and technological innovation project of double-city economic circle construction in Chengdu-Chongqing area (No.KJCX2020024), Chongqing University Innovation Research Group funding (No.CXQT20015) 
2019). Self-training algorithm is widely used, since it does not need to consider initial assumptions of data set and is easy to be implemented. Self-training algorithm contains two steps. (a) A small amount of labeled samples are utilized to train an initial classifier. (b) The unlabeled samples with the highest confidence are selected and are predicted by the classifier, and then they are added into the training set. The two steps are repeated until the stop condition is reached.

How to improve the performance of self-training algorithm has been discussed and studied by many scholars in recent years. Gan et al.(2013) developed semi-supervised fuzzy C-means algorithm (SFCM) to find unlabeled samples which has strong discriminant ability in the local data structure, and unlabeled samples are relabeled through fuzzy C-means algorithm. However, the algorithm does not consider the global structure information of data set and can't obtain spatial structure on non spherical distribution data. Thus, Wu et al.(2018) proposed a self-training algorithm based on density peaks of data (STDP). The density peaks clustering (DPC) algorithm is used to discover the spatial structure of data set, which helps the self-training algorithm to relabel representative unlabeled samples.

Although STDP considers the global spatial structure of data sets, it will easily produce some mislabeled samples during the training process. If the mislabeled samples are added to the training data set, it will ultimately reduce the classification accuracy of the classifier(Wei $\mathrm{Z}$ et al. $\underline{2013}$ ). Thus, many self-training algorithms based on the local noise filters have been proposed, such as self-training based on density peaks clustering and cut edge weight statistic (STDPCEWS) (Wei D et al. 2020), self-training method based on density peaks clustering with extended parameter-free local noise filter (STDPNF) (Li J et al. 2019) and multi-label self-training with editing (MLSTE) (I. Triguero et al. 2014 ). STDPCEWS identifies mislabeled samples by cutting edges weight statistic. MLSTE adopts edited nearest neighbor (ENN) to filter out noisy samples while STDPNF uses natural neighbors to remove noisy samples. Despite these local noise filters can improve the classification accuracy of the classifiers, their main technical defects are as follows:

(a) Current local noise filters only consider the spatial distribution of the nearest neighbors within one class. Considering the different distribution of samples in different classes, local noise filters need different $k$ nearest neighbors for different classes.

(b) Local noise filters based on $k$ nearest neighbors are impacted by noise samples, especially when the size of surrounding labeled samples is small. Actually, when $k$ value is very small, the local noise filters may ignore important nearest neighbors. Conversely, if $k$ value is large, the local noise filters will be subjected to relabeled samples with the error class. Thus, it is unreasonable for ENN to utilize a fixed value $k$ to detect relabeled samples. Therefore, an adaptive selection rule is quite significant and necessary.

(c) The decision rule of current local noise filters is majority rule, which is quite imprecise. Majority rule does not take effect when mislabeled samples are located in the overlapping areas of different classes. Thus, closer nearest neighbor contributes more to detection, and it acquires more voting weights(Mateos-Garci'a et al. 2016).

Based on the above analysis, a novel globally adaptive multi-local noise filter using harmonic mean distance(GAMLNF) is proposed. GAMLNF uses the idea of the local mean-based $k$ nearest neighbor(LMKNN) (Mitani Y et al. 2006). Each class finds its correspondingly local average vector, which can take the spatial distribution of the nearest neighbors within each class into account (Gou $\mathbf{J}$ et al. 2014). Firstly, GAMLNF utilizes globally adaptive nearest neighbor selection rule (Pan Z et al. 2020), which calculates $r$ globally nearest neighbors, and GAMLNF dynamically finds out $k_{i}$ local nearest 
neighbors of each class. Secondly, $k_{i}$ multi-local mean vectors are calculated on the basis of $k_{i}$ local nearest neighbors, and the sole pseudo mean vector is calculated, which effectively reduces the influence of mislabeled samples. Finally, GAMLNF calculates the distance between pseudo mean vector of each class and unlabeled samples by harmonic mean distance (Pan Z et al. 2017a, $\underline{\text { b }}$ ). Naturally, GAMLNF can consider the influence of spatial distribution in each class. The strategies enable GAMLNF to improve the ability of detecting mislabeled samples, and the classification accuracy can be boosted.

In order to better detect the mislabeled samples in STDP, a new self-training algorithm based on density peaks combining globally adaptive multi-local noise filter(STDP-GAMLNF) is proposed in this paper. Firstly, STDP-GAMLNF uses DPC to reveal the whole spatial structure of samples (Wu et al. 2018). Again, the unlabeled samples are relabeled according to the spatial structure. Next, by using the novel noise filter, a mislabeled sample is comprehensively detected from multiple classes, and the influence of edge samples and mislabeled samples are reduced effectively. Finally, the mislabeled samples will be removed, which will corrupt the spatial structure revealed by DPC. Space structure restoration (SSR) technology with doubly-linked list is used for storing spatial structure, and automatically connects the unlabeled "previous" samples with the nearest density peak one in the deleted samples. Thus, SSR can ensure that every unlabeled sample can be correctly relabeled.

The rest of the paper is organized as follows. Preliminaries are described in Section 2. In Section 3, STDP-GAMLNF are introduced. The comparison experiments are used to evaluate the performance of STDP-GAMLNF in Section 4. And the experimental results are discussed in Section 5. Section 6 concludes our work and future plans.

\section{Preliminaries}

In this section, notations used in this paper are first shown. Then, STDP, LMKNN and the harmonic mean distance will be described briefly.

\subsection{Notations}

In this paper, the notations are described in Table 1.

Table 1 The notations used in this paper.

\begin{tabular}{|c|c|c|}
\hline ID & Notations & $\begin{array}{l}\text { Explanations. } \\
\end{array}$ \\
\hline 1 & $\boldsymbol{T}=\left\{\left(x_{i}, y_{i}\right) \mid 1 \leq \mathrm{i} \leq n\right\}$ & $x_{i}$ is the training samples, $y_{i}$ is its corresponding label. \\
\hline 2 & $n$ & The size of data set. \\
\hline 3 & $\boldsymbol{Y}=\left\{y_{i} \mid y_{i} \in\left\{w_{i}\right\}, i=1,2 \ldots, \mathrm{m}\right\}$ & The number of class. \\
\hline 4 & $\boldsymbol{L}=\left\{l_{l}, l_{2}, \ldots, l_{u}\right\}$ & The labeled data set. \\
\hline 5 & $\boldsymbol{U}=\left\{u_{l}, u_{2}, \ldots, u_{l}\right\}$ & The unlabeled data set. \\
\hline 6 & $w_{j}$ & The $j$ th class of data set. \\
\hline 7 & $\mathrm{NN}_{\mathrm{j}}^{\mathrm{r}}$ & The nearest neighbor set for class $w_{\mathrm{j}}$ with $\mathrm{r}$ nearest neighbors. \\
\hline 8 & $m_{j}$ & The local mean vector for class $w_{j}$. \\
\hline 9 & $\operatorname{HMD}(\cdot)$ & The harmonic mean distance. \\
\hline 10 & $\operatorname{AMD}(\cdot)$ & The arithmetic mean distance. \\
\hline 11 & $d\left(x_{t}, x_{i}\right)$ & The Euclidean distance between $x_{t}$ and $x_{i}$. \\
\hline 12 & $P D$ & The probability difference between the highest probability and the lowest one. \\
\hline 13 & $\beta$ & Local density. \\
\hline 14 & $\delta$ & The nearest local density sample. \\
\hline 15 & $L_{j}$ & The labeled samples set in class $w_{j}$ \\
\hline 16 & order & The spatial structure revealed by DPC. \\
\hline 17 & $r_{i}$ & The number of nearest neighbors in class $w_{j}$. \\
\hline
\end{tabular}

\subsection{STDP algorithm}


STDP algorithm first uses DPC to reveal the whole spatial structure of the samples (Wu et al. 2018). DPC is a density-based clustering algorithm that can discover the global spatial structure of data and automatically find the number of clusters. DPC is based on the two assumptions:

(a) The highest density sample in a cluster is the center sample of the cluster.

(b) There is a large distance between centroid samples.

Based on two assumptions, DPC first calculates the local density $\beta_{i}$ and the nearest local density sample $\delta_{i}$ for each sample $x_{i}$. The spatial structure of the data can be found by assigning $x_{i}$ to its corresponding sample $\delta_{i}$, where $\beta_{i}$ and $\delta_{i}$ can be computed as:

$$
\begin{aligned}
& \beta_{i}=\sum_{j} \mathrm{X}\left(d_{i j}-d_{c}\right) \\
& \mathrm{X}(x)= \begin{cases}1, & x<0 \\
0, & x \geq 0\end{cases} \\
& \delta_{i}= \begin{cases}\min _{J: \beta_{i}<\beta_{j}}\left(d_{i j}\right), \text { others } \\
\max _{j}\left(d_{i j}\right), & \forall j, \beta_{i} \geq \beta_{j}\end{cases}
\end{aligned}
$$

STDP uses the global spatial structure revealed by DPC to select representative unlabeled samples for relabeling. The relabeling process is divided into two steps. Step 1 is iteratively to select and relabel all the unlabeled "next" samples of the labeled samples. Step 2 is iteratively to select and relabel all the unlabeled "previous" samples of the labeled samples. A general framework for STPD is shown in Fig. 1.

Step 1

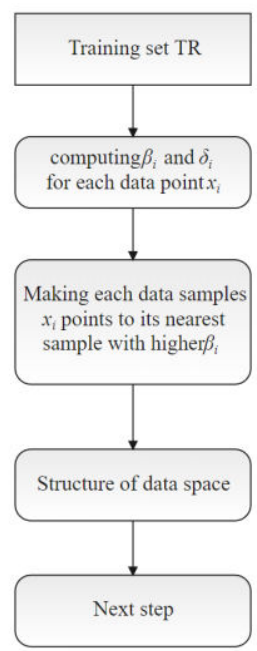

Step 2

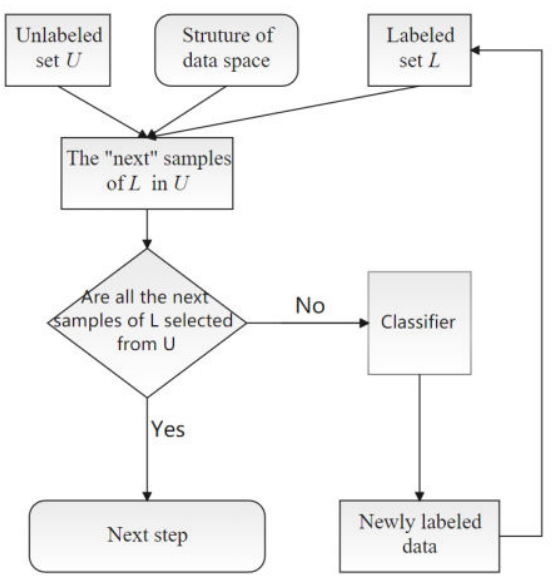

Step 3

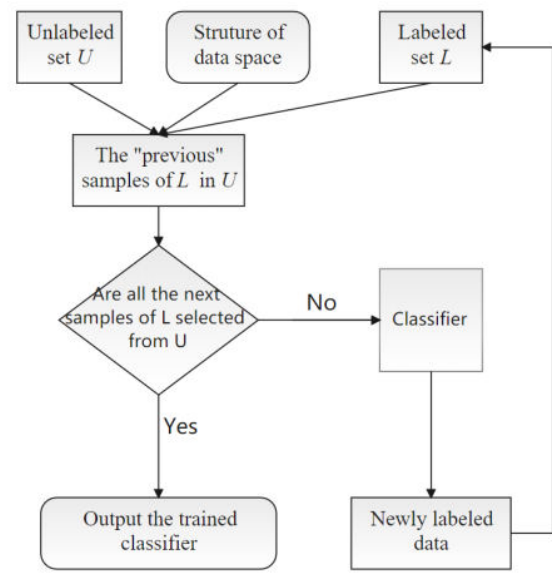

\subsection{LMKNN algorithm}

Fig. 1 The framework of STDP.

LMKNN algorithm is a very effective and simple classification algorithm in supervised learning (Mitani Y et al. 2006). It proposes a local mean vector and distance decision rule and considers the spatial distribution of the nearest neighbors of each class. The algorithm is immune to outliers, and can improve the accuracy of classification.

Suppose that $\boldsymbol{L}_{j}=\left\{\left(x_{i}, y_{i}\right) \mid\left(1 \leq \mathrm{i} \leq \mathrm{n}_{\mathrm{j}}\right)\right\}, \boldsymbol{L}_{j}$ is labeled samples set in class $w_{j}, \mathrm{U}_{\mathrm{j}=1}^{w} L_{j}=L$, and the parameter $r$ represents the number of neighbors in each class. The unlabeled sample $x^{*} \in \mathrm{R}^{\mathrm{d}}$ would be relabeled as following: 
Self-training algorithm based on density peaks combining globally adaptive multi-local noise filter

First of all, $r$ samples nearest to $x_{i}$ in $\boldsymbol{L}_{j}$ are calculated by local nearest neighbor(LNN) rule, which is given in Algorithm $1 . \mathrm{NN}_{\mathrm{j}}^{\mathrm{r}}=\left\{\left(\mathrm{x}_{i}, y_{i}\right) \mid y_{i}=w_{\mathrm{j}}, 1 \leq i \leq r\right\}$ represents the $r$ samples point nearest to $x_{i}$ in the class $w_{\mathrm{j}}$, then the $r$ samples are sorted in ascending order.

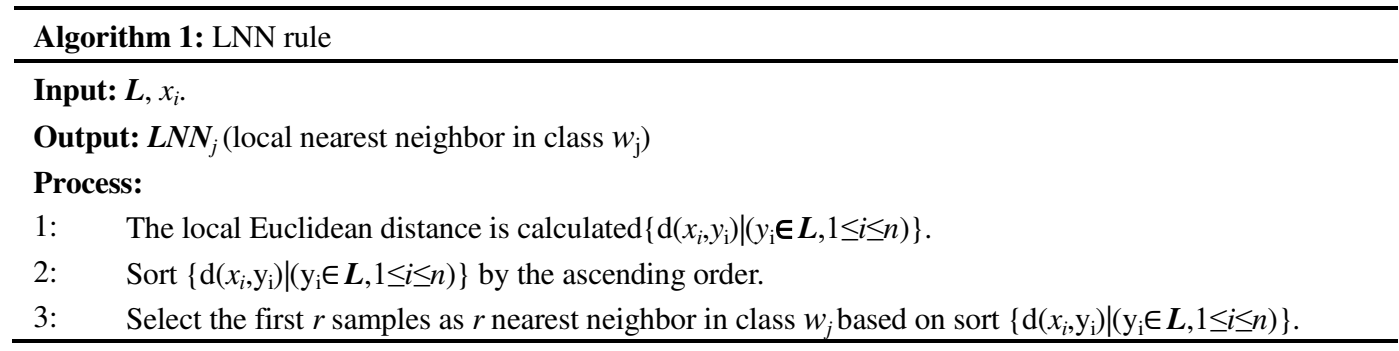

After that, the local mean vector $m_{j}^{r}$ is calculated from the $r$ nearest samples in each class

$$
m_{j}^{r}=\frac{1}{r} \sum_{i=1}^{r} y_{i}, y_{i} \in \mathrm{NN}_{j}^{r}
$$

Finally, the local mean vector closest to unlabeled sample $x_{i}$ is obtained, the class of $x_{i}$ is the class of the local mean vector, which is calculated by Eq. (4)

$$
w_{c}=\arg \min _{w_{j}} d\left(x, m_{j}^{r}\right)
$$

where Euclidean distance is used to find the local mean vector nearest to $x$.

\subsection{The harmonic mean distance}

The harmonic mean distance is defined as $\operatorname{HMD}(\cdot)$ ( $\operatorname{Pan} \mathrm{Z}$ et al. 2017a,$\underline{b}$ ), which is used to measure the distance between the unlabeled sample $x$ and $n$ local mean vector of labeled samples in class $w \operatorname{LAM}_{w}(x)=\left\{m_{w}^{i} \mid 1 \leq i \leq n\right\}$. The harmonic mean distance $\operatorname{HMD}\left(\mathrm{x},\left\{m_{w}^{i}\right\}_{i=1}^{n}\right)$ is defined as

$$
\operatorname{HMD}\left(\mathrm{x},\left\{\mathrm{m}_{w}^{\mathrm{i}}\right\}_{\mathrm{i}=1}^{\mathrm{n}}\right)=\frac{n}{\sum_{i=1}^{n} \frac{1}{\mathrm{~d}\left(\mathrm{x}, \mathrm{m}_{w}^{\mathrm{i}}\right)}}
$$

In order to explain the difference between harmonic average distance and arithmetic mean distance, the proof is given.

The arithmetic mean distance is used to measure the distance between an unlabeled sample $x$ and $n$ local mean vector set in class $w \operatorname{LAM}_{w}(x)=\left\{m_{w}^{i} \mid 1 \leq i \leq n\right\}$, which is denoted as $\operatorname{AMD}\left(x,\left\{m_{w}^{i}\right\}_{i=1}^{n}\right)$, as in

Eq. (6), the value of $\mathrm{d}\left(x, m_{w}^{i}\right)$ is represented $\frac{\partial A M D\left(x,\left\{m_{\mathrm{w}}^{i}\right\}_{i=1}^{n}\right)}{\partial d\left(x, m_{\mathrm{w}}^{i}\right)}$. Eq. (7) can prove that $\frac{\partial A M D\left(x,\left\{m_{\mathrm{w}}^{i}\right\}_{i=1}^{n}\right)}{\partial d\left(x, m_{\mathrm{w}}^{i}\right)}=\frac{1}{n}$, which can prove that the distance between the unlabeled sample $x$ and the local average vector has the same weight, so it can not give more weight to the nearest local average vector.

$$
\operatorname{AMD}\left(\mathrm{x},\left\{\mathrm{m}_{w}^{\mathrm{i}}\right\}_{\mathrm{i}=1}^{\mathrm{n}}\right)=\frac{\sum_{i=1}^{n} \mathrm{~d}\left(\mathbf{m}_{w}^{i}\right)}{n}
$$




$$
\frac{\partial A M D\left(x,\left\{m_{w}^{i}\right\}_{i=1}^{n}\right)}{\partial d\left(x, m_{w}^{i}\right)}=\frac{\partial\left[\frac{\sum_{\mathrm{i}=1}^{n} d\left(x, m_{w}^{i}\right)}{n}\right]}{\partial d\left(x, m_{w}^{i}\right)}=\frac{\partial\left[\sum_{i=1}^{n} d\left(x, m_{w}^{i}\right)\right]}{\partial d\left(x, m_{w}^{i}\right)} \times \frac{1}{n}=\frac{1}{n}
$$

Similarly, Eq. (8) can prove that $\frac{\partial H M D\left(x,\left\{m_{w}^{i} n_{i=1}^{n}\right)\right.}{\partial d\left(x, m_{w}^{i}\right)}$ is inversely proportional to the value of $\mathrm{d}^{2}\left(x, m_{w}^{i}\right)$. Compared with the arithmetic average distance, the harmonic average distance will pay more attention to the local average vector of the labeled samples which is the closet to the unlabeled sample $x$.

$$
\begin{aligned}
\frac{\partial \mathrm{H} M D\left(x,\left\{m_{w}^{i}\right\}_{i=1}^{n}\right)}{\partial d\left(x, m_{w}^{i}\right)}= & \frac{\partial\left[\frac{n}{\sum_{i=1}^{n} \frac{1}{d\left(x, m_{w}^{i}\right)}}\right]}{\partial d\left(x, m_{w}^{i}\right)}=\mathrm{n} \times\left(\frac{1}{\partial d\left(x, m_{w}^{i}\right) \times \sum_{i=1}^{n} \frac{1}{d\left(x, m_{w}^{i}\right)}}\right)^{2} \\
& =\frac{\mathrm{H} M D^{2}\left(x,\left\{m_{w}^{i}\right\}_{i=1}^{n}\right)}{n \times d^{2}\left(x, m_{w}^{i}\right)}=\frac{1}{\mathrm{n}} \times \frac{\mathrm{H} M D^{2}\left(x,\left\{m_{w}^{i}\right\}_{i=1}^{n}\right)}{d^{2}\left(x, m_{w}^{i}\right)}
\end{aligned}
$$

\section{The proposed algorithm}

Fig. 2 shows the framework of STDP-GAMLNF, which is mainly divided into three parts. The first part is to discover the spatial structure of data set via DPC. The second part is to relabel the unlabeled "next" samples of labeled samples according to the spatial structure, and use GAMLNF to detect mislabeled samples. If it is detected as a mislabeled sample, SSR is used to repair the spatial structure to ensure that each unlabeled sample can be relabeled. Otherwise, the unlabeled sample is added to $\boldsymbol{L}$. Finally, the unlabeled "previous" samples of the labeled samples are relabeled according to the updated spatial structure. GAMLNF is used to detect mislabeled samples, and SSR is used to repair the spatial structure. After that, all unlabeled samples are relabeled as labeled samples, and the labeled samples can train a better supervised classifier.

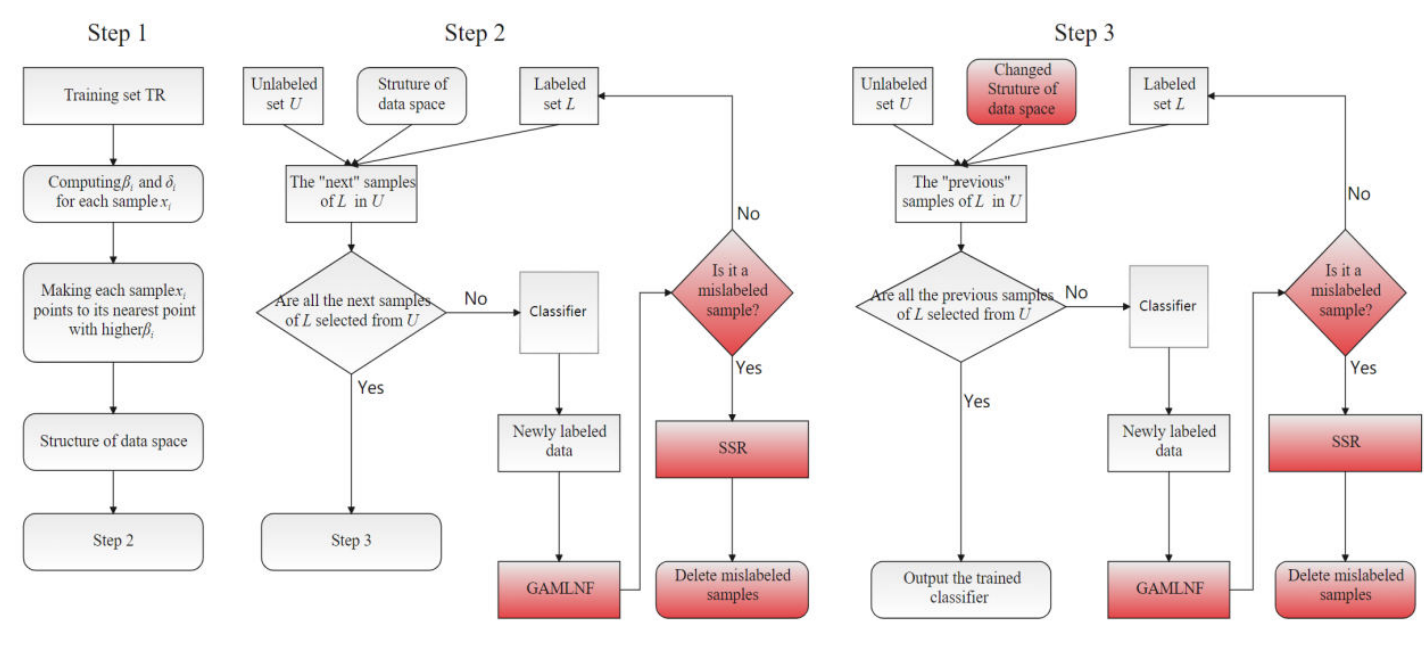

Fig. 2 The framework of STDP-GAMLNF.

\subsection{Globally adaptive multi-local noise filter using harmonic mean distance}


In the semi-supervised learning environment, GAMLNF can adaptively find the appropriate $k$ parameters to detect and delete the mislabeled samples, which can effectively improve the algorithm accuracy.

Inspired by the improved algorithm GAKNN of LMKNN summarized in 2.3, we first use GANN to find the neighbors of labeled sample around relabeled samples. Specifically, the labeled sample set $\boldsymbol{L}$ has $m$ classes, GANN can adaptively find $r_{i}$ nearest neighbors in each class around the relabeled sample $x_{i}$, as shown in Algorithm 2.

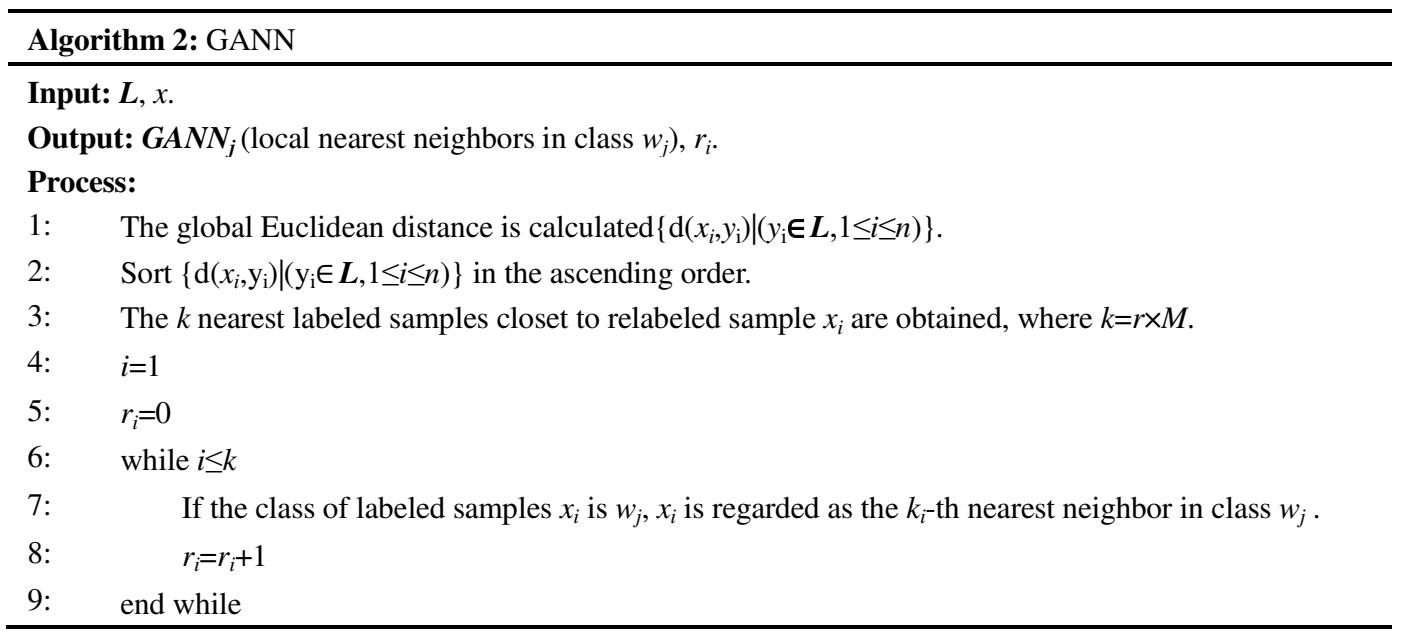

When GANN adaptively finds $r_{i}$ nearest neighbors in each class, the multi-local average vector $\operatorname{MLMV}_{\mathrm{j}}=\left\{\mathrm{m}_{\mathrm{j}}^{\mathrm{s}} \mid 1 \leq s \leq k\right\}$ in each class can be calculated, where $\mathrm{m}_{j}^{s}$ is the local average vector, and is calculated by first $s$ nearest neighbor in class $w_{j}$, its calculation is shown in Eq. (9).

$$
\mathrm{m}_{\mathrm{j}}^{\mathrm{s}}=\frac{1}{s} \sum_{i=1}^{s} y_{i}, y_{i} \in \mathrm{GANN}_{j}^{\mathrm{s}}
$$

Closer local average vector contributes more to detection, so it acquires more voting weights. Thus, $\operatorname{HMD}\left(x_{i}, m_{j}^{r}\right), m_{j}^{r} \subseteq \mathrm{MLMV}_{\mathrm{j}}^{r}$ of Eq. (5) is used to calculate the pseudo average vector $m_{j}^{*}$. In particular, if there isn't any neighbors in class $w_{j}$, then $\mathrm{d}\left(x_{i}, w_{j}\right)=\infty$. Lastly, the closest pseudo average vector to the relabeled sample $x_{i}$ is calculated by the Euclidean distance. If the relabeled sample is not in accordance with the class of $m_{j}^{*}$, the relabeled sample is considered as a mislabeled sample and is deleted from $\boldsymbol{U}$. Otherwise, it is added to the $\boldsymbol{L}$ set. GAMLNF is shown in Algorithm 3.

The computational complexity of GAMLNF mainly occurs in the search of global nearest neighbor (Stage 1), the pseudo average vector calculation (Stage 2) and the Euclidean distance calculation (Stage 3). In Stage 1, GAMLNF obtains $k$ global nearest neighbors from $n$ labeled samples according to Algorithm 2. Therefore, it is easy to calculate the computational complexity as $O(n k)$.

In Stage 2, GAMLNF first needs to calculate $m \times r$ local average vectors, and then calculate $m$ pseudo average vectors through the harmonic average distance. Hence, it is easy to see that the computational complexity is $O(m r)$ according to Algorithm 3.

In Stage 3, GAMLNF obtains the closest pseudo-average vector to $x$ from the $m$ pseudo-average vectors and its complexity is $O(m)$. 


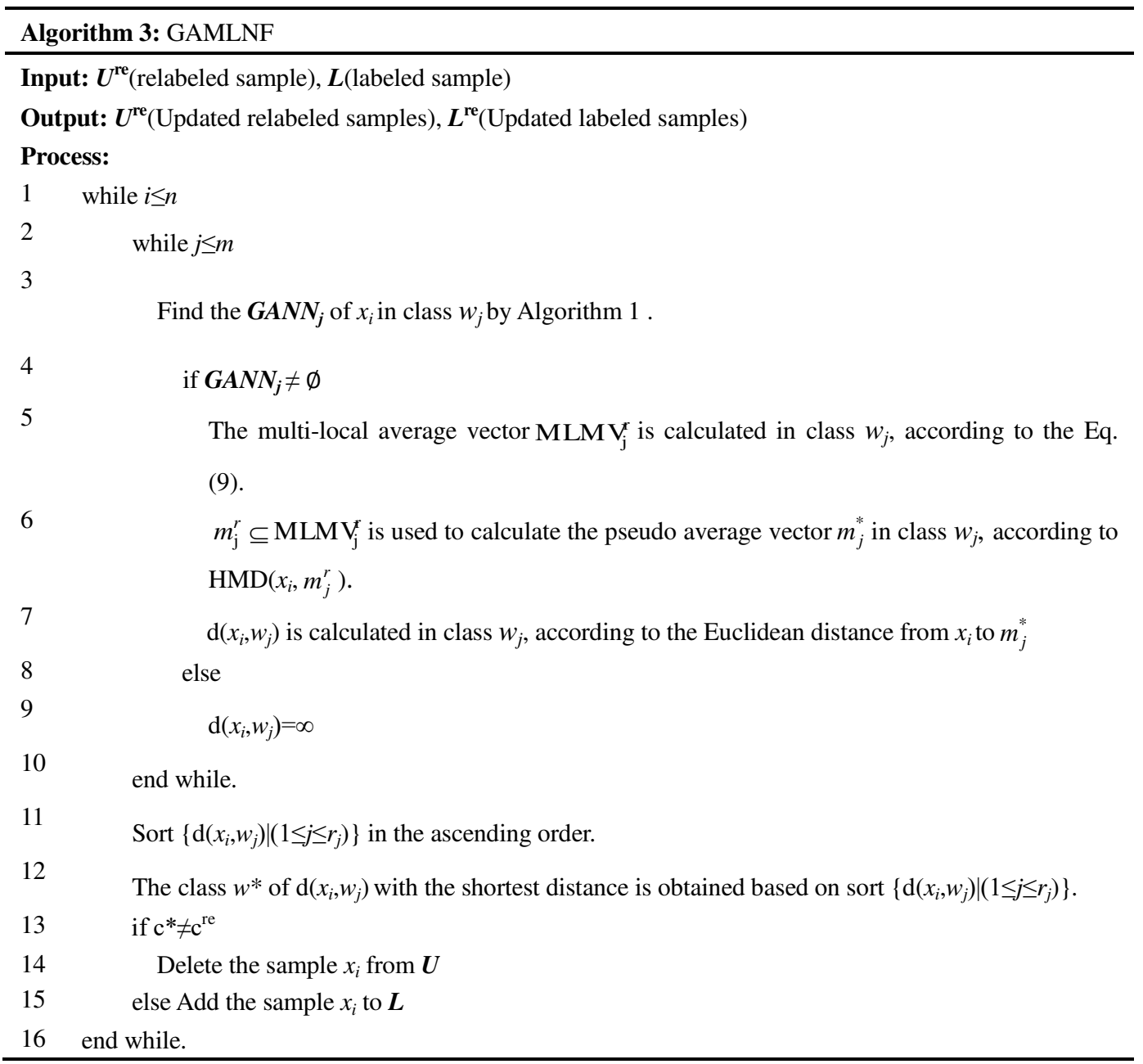

\subsection{Space structure restoration}

GMLNF will be used to detect and delete the mislabeled samples. However, it will corrupt the spatial structure disclosed by DPC. As shown in Fig. 3, the circles and triangles represent real class $w_{1}$ and class $w_{2}$, the green and blue represent labeled samples as $w_{1}$ and $w_{2}$, colorless symbols represent unlabeled samples. As shown in Fig. 3(a), each sample will be designated to nearest and highest local density sample in the spatial structure disclosed by DPC. The unlabeled "next" samples will be relabeled by the spatial structure. However, as shown in Fig. 3(b), when the unlabeled "next" samples $x_{2}$ and $x_{3}$ are designated to an relabeled sample $x_{1}$, and there are no "previous" other samples between $x_{2}$ and $x_{3}$. If $x_{1}$ is mislabeled sample and $x_{1}$ is deleted by the GMLNF, the spatial structure will be corrupted, $x_{2}$ and $x_{3}$ can not be relabeled.

Accordingly, SSR algorithm is proposed. As shown in Fig. 3(c), the doubly-linked list structure is used to store the spatial structure. When $x_{1}$ is deleted by the noise filter, the nearest highest local density sample $x_{4}$ is obtained by the doubly-linked list structure, then $x_{2}$ and $x_{3}$ are designated to $x_{4}$. When the unlabeled sample $x_{4}$ is relabeled, the unlabeled samples $x_{2}$ and $x_{3}$ will have the chance to be relabeled. SSR algorithm is shown in Algorithm 4. 
Self-training algorithm based on density peaks combining globally adaptive multi-local noise filter

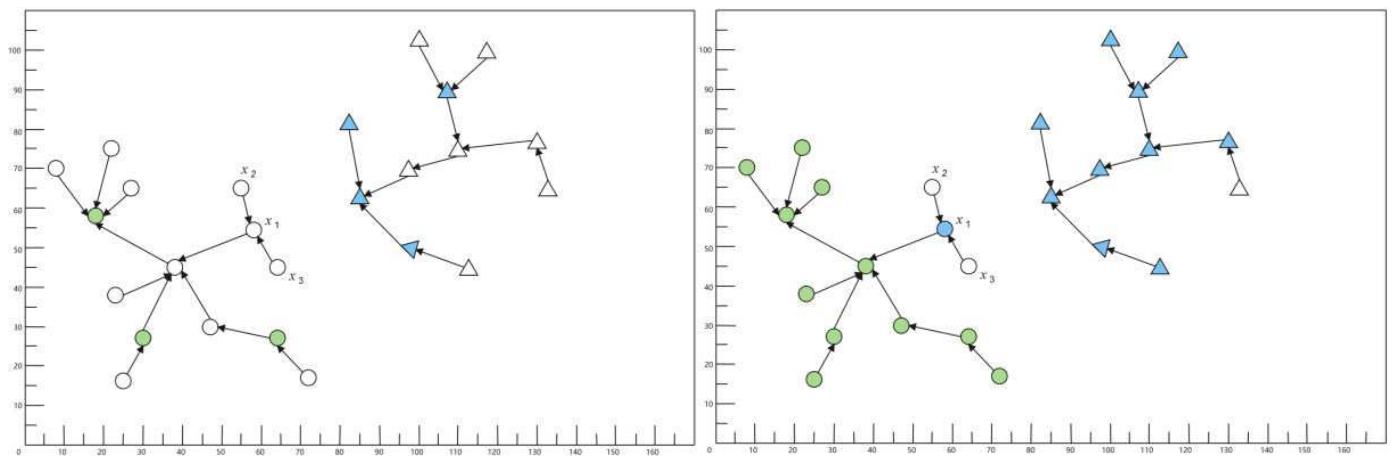

(a)The spatial structure disclosed by DPC

(b)The spatial structure is corrupted

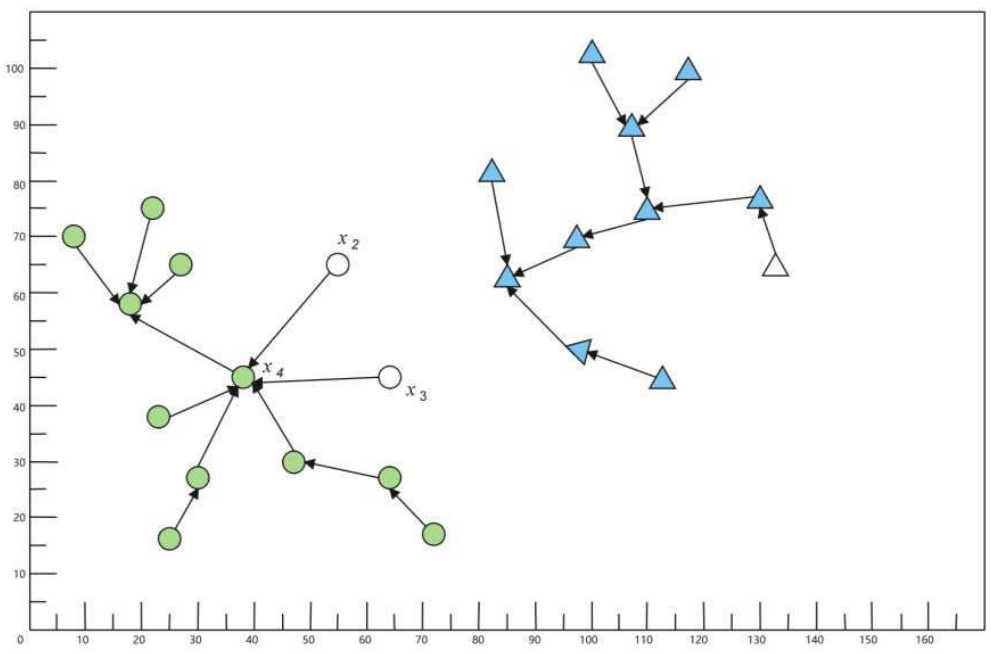

(c)The spatial structure is repaired

Fig. 3 Space structure restoration step diagram

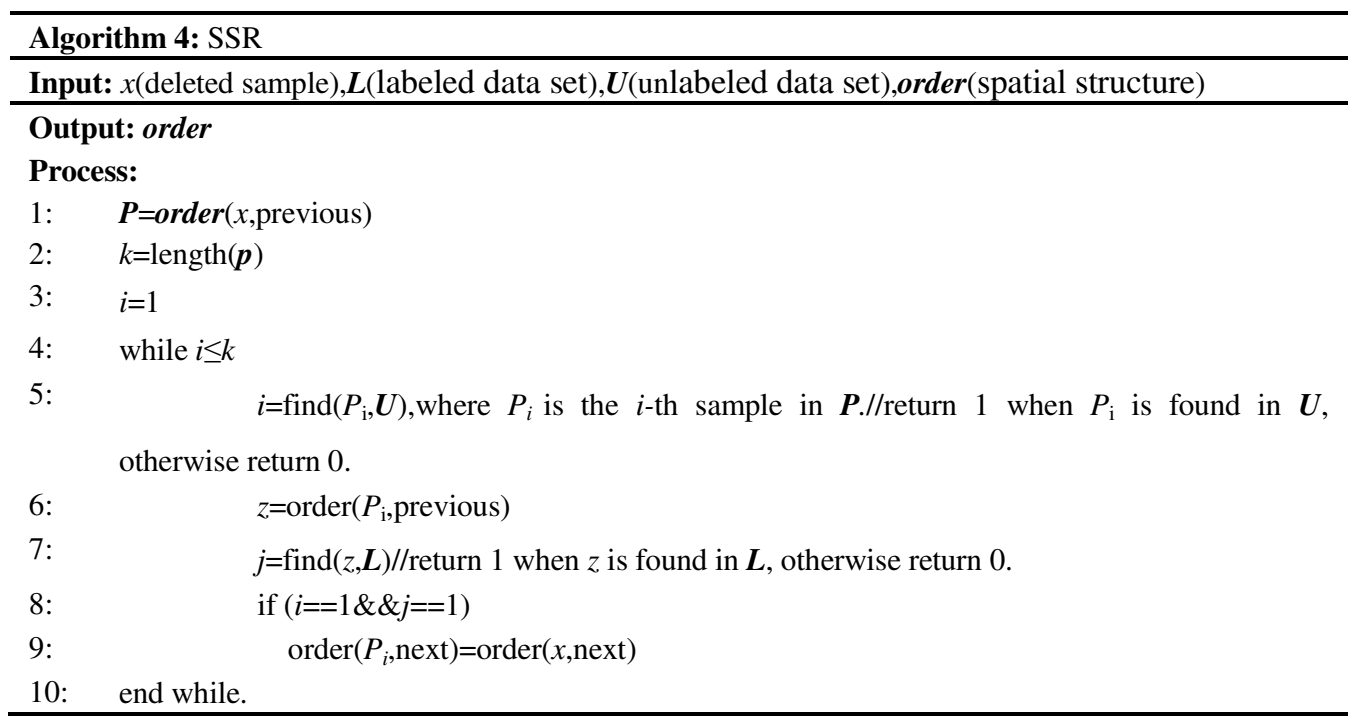




\subsection{Self-training algorithm based on density peaks combining globally adaptive multi-local noise filter}

The whole algorithm consists of five steps. At Step 1-Step 4, DPC algorithm is used to reveal the spatial structure of the data set. The spatial structure is stored in the form of doubly-linked list. At the same time, $\boldsymbol{L}$ is used to train the initial classifier C. Step 5-Step 11 are a process of iteratively training classifier C. Firstly, according to the order of spatial structure, the unlabeled "next" sample of a labeled sample is relabeled by the classifier C. Then the unlabeled samples are detected by GAMLNF. SSR is applied to the mislabeled samples which may cause the corruption of spatial structure, and then the relabeled samples are added to the $\boldsymbol{L}$. Finally, the classifier C is trained on the basis of updated $\boldsymbol{L}$. Step 12-Step 18 will be iteratively training classifier $\mathrm{C}$ with operate as similar as Step 5-Step 11.

STDP-GAMLNF can not only consider the global spatial structure revealed by DPC, but also consider the spatial structure in each class by GANN algorithm. Simultaneously, GAMLNF can adaptively find $r_{i}$ local nearest neighbors from the $k$ global nearest neighbors in each class. GAMLNF adopts the harmonic average distance to give more voting weight to the nearest neighbor. Based on the above improvements, STDP-GAMLNF can quickly train an effective supervised classifier.

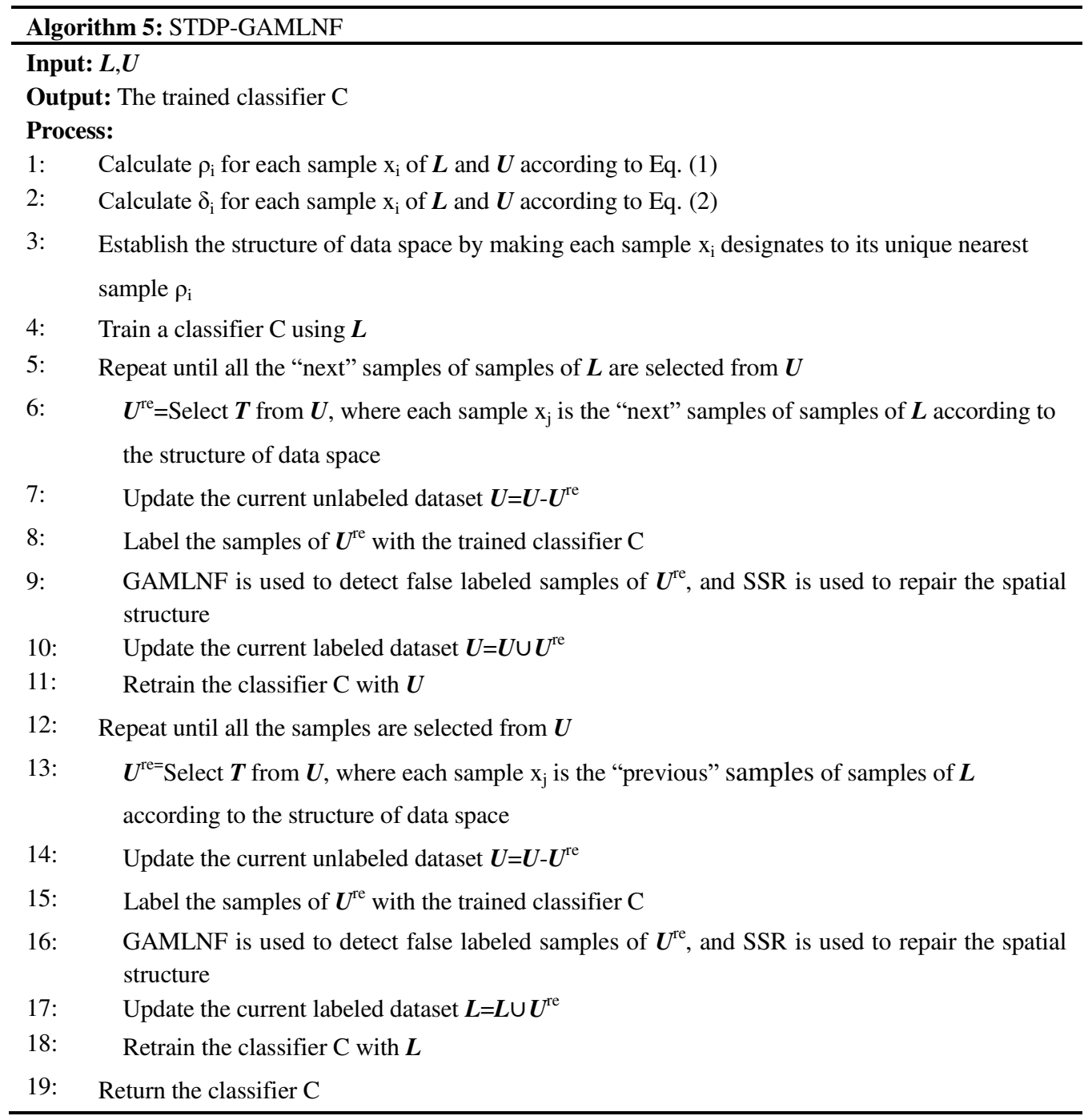




\section{Experiments}

\subsection{Data sets and setting of experiments}

We use a PC with 32G memory, Core i5 CPU and 64-bit operating system to run some experiments, in order to verify the efficiency of the proposed algorithm. Also, MATLAB2016b and PyCharm2021 are utilized to program.

In order to prove the effectiveness of STPD-GAMLNF, 18 benchmark data sets of experiments are selected from UCI (Asuncion A 2007) repositories. The data sets are described in Table 2.

Table 2 The descriptions of UCI data sets.

\begin{tabular}{cccccc}
\hline ID & Data sets & Size & Attributes & Class & Abbreviation \\
\hline 1 & Australian_Credit_Approval & 690 & 2 & 2 & ACA \\
2 & biodegradation & 1055 & 41 & 2 & BIO \\
3 & Blood_Transfusion_Service_Center & 748 & 4 & 2 & BTSC \\
4 & Avila & 20867 & 12 & 12 & AVI \\
5 & Contraceptive_Method_Choice_Data_ & 1473 & 9 & 3 & CMCDS \\
& Set & 2000 & 50 & 2 & GAU \\
6 & Gauss50 & 1000 & 24 & 2 & GER \\
7 & german & 2310 & 19 & 7 & IS \\
8 & Image_segmentation & 583 & 10 & 2 & ILPD \\
9 & Indian_Liver_Patient_Dataset & 961 & 5 & 2 & MM \\
10 & mammographic_masses & 2130 & 19 & 7 & SEG \\
11 & Segmentation & 4601 & 57 & 2 & SPA \\
12 & spambase & 958 & 9 & 2 & TTTE \\
13 & Tic_Tac_Toe_Endgame & 1353 & 9 & 3 & WP \\
14 & Website_Phishing & 5000 & 21 & 3 & WAV \\
15 & WaveForm & 2000 & 7 & 4 & WIL \\
16 & Wireless_Indoor_Localization & 8800 & 2 & 2 & BAN \\
17 & Banana & 15534 & 16 & 26 & LET \\
18 & letter &
\end{tabular}

The training set is randomly divided into the labeled sample set $\boldsymbol{L}$ and the unlabeled sample set $\boldsymbol{U}$. Four experiments are used to verify the effectiveness of the proposed algorithm. 10-fold cross-validation is used in all the experiments. Brief descriptions are listed as follows:

(a) Experiment 1 is a comparative experiment between STDPGAMLNF and other representative algorithm. Mean accuracy, variance, ROC, AUC work as evaluation indexes, and the ratio of $\boldsymbol{L}$ is set at $20 \%$. The CART is selected as the base classifier.

(b) Experiment 2 compares the effect of the noise filter with the representative noise filter in STDP. Kappa coefficient is used as evaluation index, and the ratio of $\boldsymbol{L}$ is set at $20 \%$. CART and KNN are selected as the base classifier.

(c) Experiment 3 studies the influence of $k$ value on the proposed algorithm. KNN is selected as the base classifier.

(d) Experiment 4 studies the impact of the proportion of labeled samples on the noise filter. The evaluation indexes are accuracy and variance, and the ratio of $\boldsymbol{L}$ is set from $10 \%$ 90\%. The base classifier adopts KNN.

\subsection{Experiment 1: comparisons between our algorithm and existing representative work.}


This experiment compares our algorithm with some representative algorithms to prove the effectiveness of STDPGAMLNF. Mean accuracy, variance, ROC, AUC will be used to prove the ability of our noise filter to detect mislabeled samples in this experiment. The ROC curve for multi-classification is calculated as:

Suppose the number of test samples is $n$ and that of class is $w$. After the training, the probability of each test sample in each class is calculated, and matrix $\boldsymbol{P}$ with $w \times n$ is obtained. Each row represents the probability value of a test sample in each class (sorted by class label). Correspondingly, the label of each test sample is converted into a one-hot encoding form, and each position is used to label whether it belongs to the corresponding class, which can also get label matrix $\boldsymbol{L}$.

Under each class, the probability of $n$ test samples of the class (column in matrix P) can be calculated. Therefore, according to each corresponding column in the probability matrix $\boldsymbol{P}$ and the label matrix $\boldsymbol{L}$, the False Positive Rate (FPR) and the True Positive Rate (TPR) under each threshold can be calculated to draw a ROC curve. In this way, $w$ ROC curves can be drawn. At last, average the $w$ ROC curves to get the final ROC curve and AUC value.

Comparison algorithms and our algorithm are shown in Table 3. And $d c$ parameter of the proposed algorithm will be discussed in detail in STDP (2018), so $d c$ of the algorithms is set as recommended in STDP. The introduction of the comparison algorithms are as follows:

(a) Self-training with editing (SETRED) (Li M et al. 2005) combines CEWS to filter out noisy samples via self-training algorithm.

(b) Multi-label self-training with editing (MLSTE) (Wei et al. 2013) use ENN to detect noisy samples by self-training algorithm. Although MLSTE is applied to multi-label tasks, it can also be applied to the single-label tasks.

(c) Self-training with SFCM (STSFCM) (Gan et al. 2013) is a clustering algorithm, which is used to guide self-training method to train an effective classifier.

(d) Self-training with DPC (STDP) (Wu D et al. $\underline{2018}$ ) integrate the structure of feature space revealed by DPC into the self-training process to train an effective classifier.

(e) STDP integrated by parameter-free local noise filter (STDPNF) (Li J et al. 2019) is parameter-free and can remove mislabeled samples by exploiting the information of both labeled data and unlabeled data.

As shown in Table 4, the accuracy of STDP-GAMNF is better than that of the comparison algorithms. Compared with STDPNF, it can accurately detect mislabeled samples better and can consider the spatial structure within each class. 12 out of 18 data sets' average accuracy of STDP-GAMNF is better than that of the comparison algorithms, and good stability can be seen from the variance. However, in AVI and MM data sets, the average accuracy of STDP-GAMNF is lower than that of STDP. The distribution of the two data sets is totally even, and the correctly marked samples are deleted during the noise filter detection, causing massive samples reductions in $\boldsymbol{L}$ set. Finally, it leads to a decrease in the average accuracy rate. Meanwhile, according to Table 5, 9 out of 18 data sets' AUC value are improved compared with STDP, which proves that STDPGAMLNF can not only improve the average accuracy but also increase the AUC value to get better classification effect. Figure 4 and Figure 5 respectively show the ROC of the two-class data set BIO and the multi-class data set CMCDS. It can be seen that STDP-GAMNF can increase ROC in each class and solve the data skew problem. 
Self-training algorithm based on density peaks combining globally adaptive multi-local noise filter

Table 3 Comparison algorithms and parameters in experiment 1.

\begin{tabular}{cll}
\hline Abbreviation & \multicolumn{1}{c}{ Algorithms } & \multicolumn{1}{c}{ Parameters } \\
\hline SETRED & Self-training with editing & $\theta=0.1$ \\
MLSTE & Multi-label self-training with editing & $k=10$ \\
STSFCM & Self-training with SFCM & Threshold $\varepsilon_{1}=1 / n$ ( $n$ is the number of classes $)$ \\
STDP & Self-training with DPC & $P_{a}=2$. Please refer to the original literature \\
STDPNF & STDP combining PNF & $P_{a}=2$. \\
& & $P_{a}=2 . r=k \times n(n$ is the number of classes $)$ \\
\hline
\end{tabular}

Table 4 Experimental results (MCA \pm STD) of comparison algorithms.

\begin{tabular}{|c|c|c|c|c|c|c|}
\hline Data sets & ST_SFCM & MLSTE & SETRED & STDP & STDPNF & Ours \\
\hline ACA & $83.91 \pm 5.48$ & $67.82 \pm 4.77$ & $68.55 \pm 8.02$ & $83.91 \pm 6.08$ & $83.91 \pm 6.08$ & $84.05 \pm 5.87$ \\
\hline $\mathrm{BIO}$ & $82.93 \pm 2.89$ & $71.07 \pm 5.46$ & $74.39 \pm 5.36$ & $83.03 \pm 2.74$ & $84.25 \pm 2.76$ & $83.78 \pm 2.93$ \\
\hline BTSC & $78.74 \pm 3.12$ & $77.01 \pm 2.53$ & $79.27 \pm 3.22$ & $79.27 \pm 2.59$ & $79.01 \pm 3.38$ & $80.21 \pm 2.94$ \\
\hline AVI & $89.83 \pm 0.59$ & $68.41 \pm 0.8$ & $70.34 \pm 0.82$ & $90.31 \pm 0.85$ & $90.02 \pm 0.72$ & $90.02 \pm 0.54$ \\
\hline CMCDS & $52.53 \pm 3.75$ & $50.57 \pm 3.75$ & $51.72 \pm 4.69$ & $54.77 \pm 4.46$ & $53.35 \pm 3.79$ & $55.66 \pm 3.15$ \\
\hline GAU & $74.9 \pm 1.85$ & $92.65 \pm 1.39$ & $88.05 \pm 2.76$ & $71.9 \pm 3.26$ & $73.95 \pm 3.1$ & $78.4 \pm 3.09$ \\
\hline GER & $71.5 \pm 3.92$ & $68.9 \pm 5.3$ & $69.1 \pm 4.12$ & $72.9 \pm 4.84$ & $73.4 \pm 4.52$ & $74.6 \pm 3.27$ \\
\hline IS & $91.99 \pm 1.06$ & $80.56 \pm 2.95$ & $87.22 \pm 1.53$ & $93.33 \pm 1.4$ & $93.76 \pm 1.31$ & $94.15 \pm 0.77$ \\
\hline ILPD & $70.15 \pm 5.16$ & $56.76 \pm 8.88$ & $67.39 \pm 7.6$ & $68.93 \pm 4.54$ & $71.35 \pm 4.77$ & $72.21 \pm 3.91$ \\
\hline MM & $83.55 \pm 2.95$ & $76.27 \pm 5.11$ & $78.98 \pm 4.32$ & $83.45 \pm 4.7$ & $83.56 \pm 4.91$ & $81.79 \pm 5.47$ \\
\hline SEG & $91.60 \pm 2.06$ & $83.63 \pm 2.36$ & $86.06 \pm 1.92$ & $93.33 \pm 1.99$ & $93.42 \pm 1.63$ & $94.19 \pm 1.54$ \\
\hline SPA & $89.28 \pm 1.46$ & $74.59 \pm 2.41$ & $74.01 \pm 2.8$ & $89 \pm 1.65$ & $89.39 \pm 1.52$ & $89.54 \pm 1.01$ \\
\hline TTTE & $69.61 \pm 4.92$ & $60.54 \pm 2.13$ & $64.08 \pm 4.56$ & $70.02 \pm 5.08$ & $69.61 \pm 4.92$ & $71.07 \pm 4.91$ \\
\hline WP & $86.98 \pm 3.02$ & $84.47 \pm 4.17$ & $84.92 \pm 4.23$ & $87.87 \pm 2.73$ & $87.57 \pm 2.52$ & $88.83 \pm 2.86$ \\
\hline WAV & $78.38 \pm 1.75$ & $80.7 \pm 1.46$ & $83.48 \pm 1.17$ & $77.98 \pm 1.55$ & $78.38 \pm 1.53$ & $79.96 \pm 1.41$ \\
\hline WIL & $93.55 \pm 1.55$ & $97.2 \pm 1.13$ & $97.7 \pm 1.03$ & $92.4 \pm 1.82$ & $94.8 \pm 1.22$ & $95.6 \pm 1.34$ \\
\hline BAN & $90.22 \pm 1.04$ & $87.79 \pm 1.19$ & $89.95 \pm 1.05$ & $90.37 \pm 0.82$ & $90.85 \pm 1.06$ & $91.62 \pm 0.90$ \\
\hline LET & $75.82 \pm 0.95$ & $75.11 \pm 0.77$ & $81.54 \pm 0.88$ & $77.28 \pm 0.94$ & $77.5 \pm 0.56$ & $81.26 \pm 0.69$ \\
\hline
\end{tabular}


Self-training algorithm based on density peaks combining globally adaptive multi-local noise filter

Table 5 Experimental results (AUC) of comparison algorithms.

\begin{tabular}{ccccccc}
\hline Data sets & ST_SFCM & MLSTE & SETRED & STDP & STDPNF & Ours \\
\hline ACA & 0.89 & 0.78 & 0.76 & $\mathbf{0 . 9 1}$ & 0.9 & 0.89 \\
BIO & 0.85 & 0.85 & $\mathbf{0 . 8 9}$ & 0.83 & 0.87 & $\mathbf{0 . 8 9}$ \\
BTSC & $\mathbf{0 . 7 9}$ & 0.78 & 0.73 & 0.74 & 0.78 & $\mathbf{0 . 7 9}$ \\
AVI & 0.96 & 0.88 & 0.89 & $\mathbf{0 . 9 6}$ & $\mathbf{0 . 9 6}$ & 0.95 \\
CMCDS & 0.65 & 0.59 & 0.6 & 0.63 & 0.67 & $\mathbf{0 . 7 1}$ \\
GAU & 0.79 & $\mathbf{0 . 9 2}$ & 0.83 & 0.75 & 0.78 & 0.84 \\
GER & $\mathbf{0 . 7 3}$ & 0.59 & 0.59 & 0.72 & 0.68 & 0.63 \\
IS & 0.96 & 0.94 & 0.96 & 0.96 & 0.97 & $\mathbf{0 . 9 8}$ \\
ILPD & 0.62 & $\mathbf{0 . 7 9}$ & 0.68 & 0.55 & 0.64 & 0.66 \\
MM & 0.81 & 0.82 & 0.84 & $\mathbf{0 . 8 6}$ & $\mathbf{0 . 8 6}$ & $\mathbf{0 . 8 6}$ \\
SEG & $\mathbf{0 . 9 8}$ & 0.94 & 0.95 & $\mathbf{0 . 9 8}$ & $\mathbf{0 . 9 8}$ & $\mathbf{0 . 9 8}$ \\
SPA & $\mathbf{0 . 9 8}$ & 0.92 & 0.91 & $\mathbf{0 . 9 8}$ & $\mathbf{0 . 9 8}$ & $\mathbf{0 . 9 8}$ \\
TTTE & $\mathbf{0 . 8 3}$ & 0.45 & 0.57 & 0.55 & 0.58 & 0.61 \\
WP & 0.87 & 0.85 & 0.80 & $\mathbf{0 . 8 8}$ & 0.87 & $\mathbf{0 . 8 8}$ \\
WAV & 0.89 & 0.91 & $\mathbf{0 . 9 2}$ & 0.88 & 0.88 & 0.89 \\
WIL & 0.97 & $\mathbf{0 . 9 8}$ & $\mathbf{0 . 9 8}$ & 0.97 & 0.97 & $\mathbf{0 . 9 8}$ \\
BAN & 0.92 & 0.92 & $\mathbf{0 . 9 5}$ & 0.94 & 0.94 & 0.93 \\
LET & 0.91 & 0.92 & $\mathbf{0 . 9 5}$ & 0.9 & 0.91 & 0.93 \\
\hline
\end{tabular}




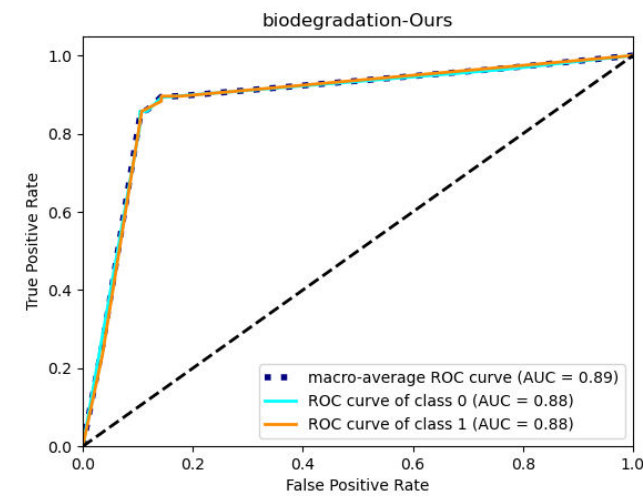

(a)STDP-GAMNF

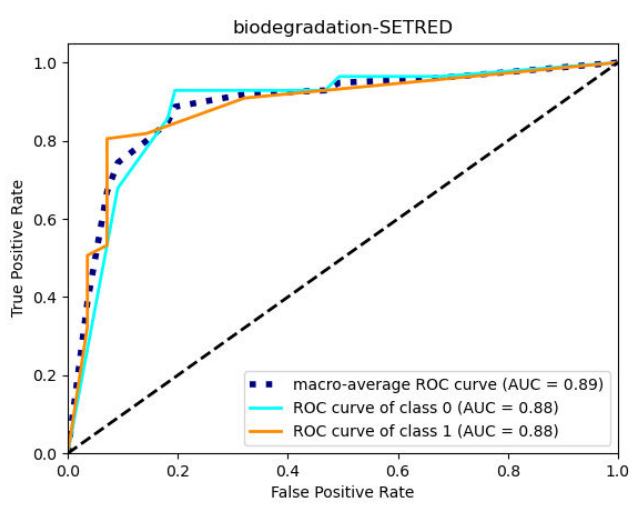

(c)SETRED

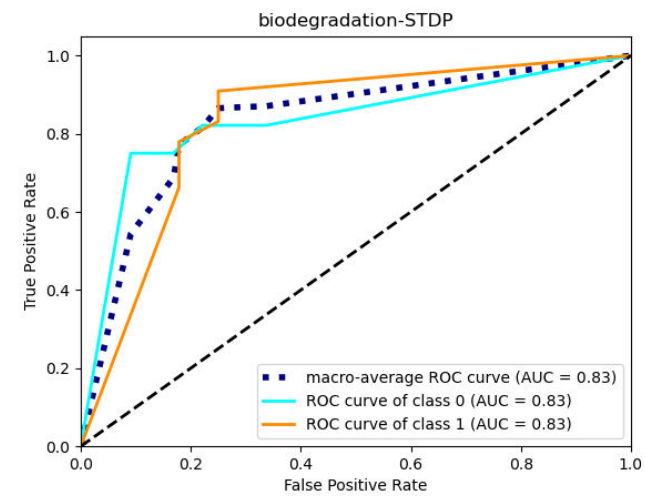

(e)STDP

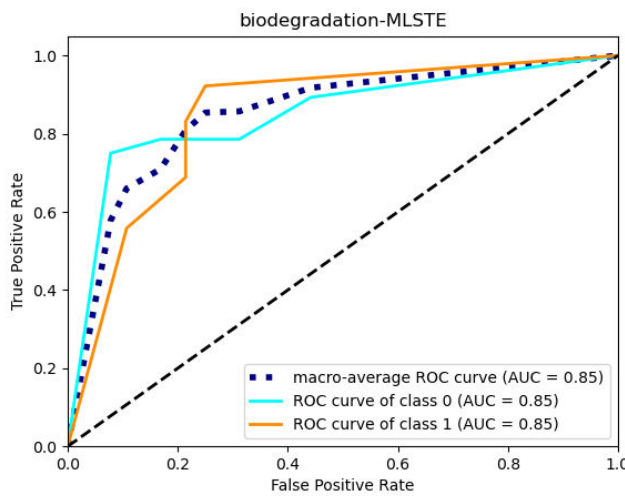

(b)MLSTE

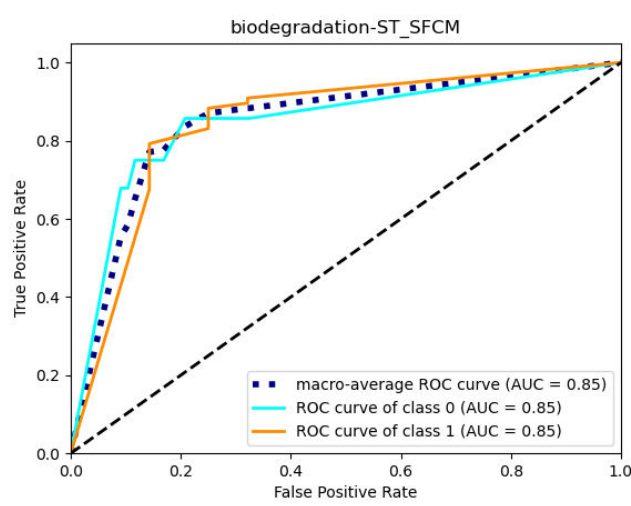

(d)ST_SFCM

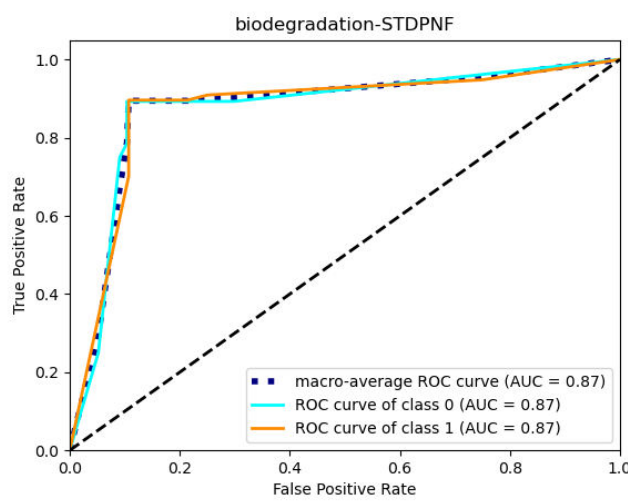

(f)STDPNF

Fig. 4 ROC of biodegradation data set 


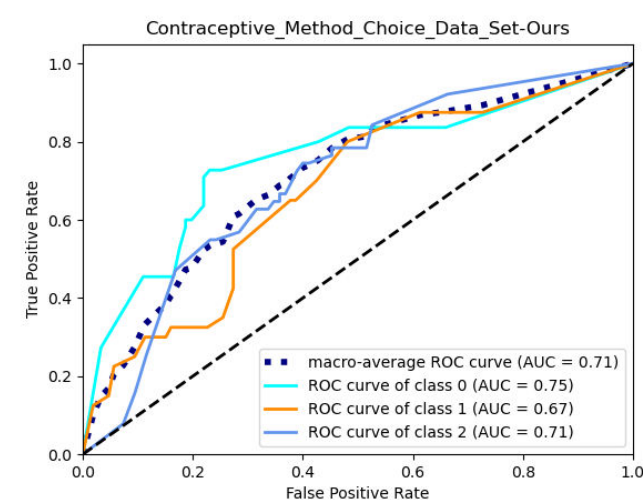

(a)STDP-GAMNF

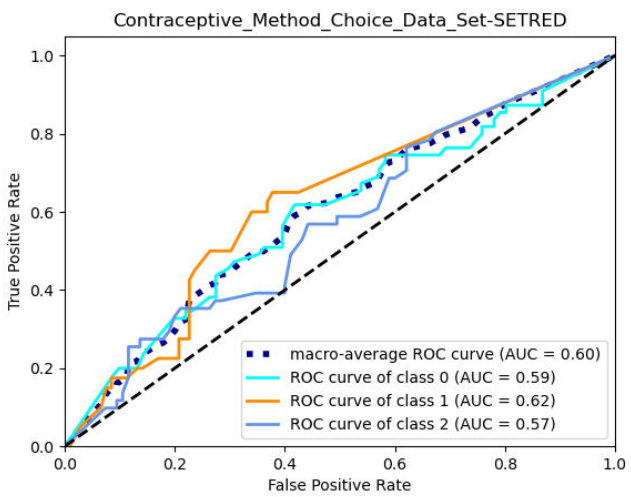

(c)SETRED

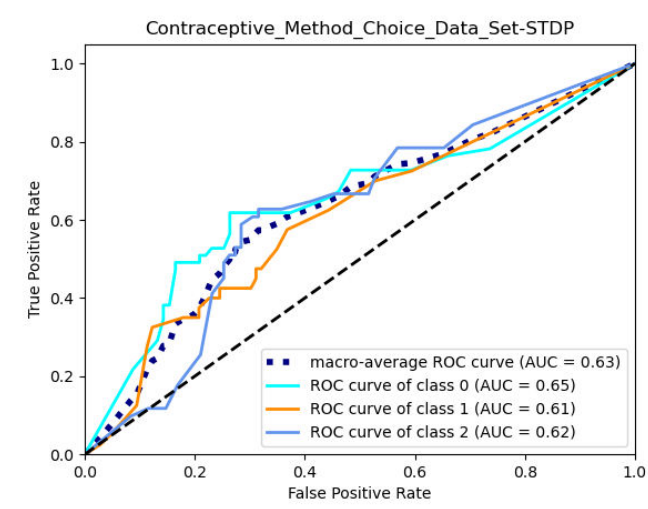

(e)STDP

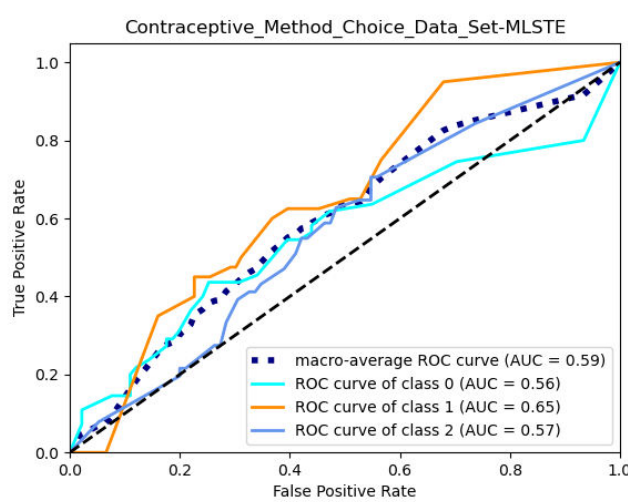

(b)MLSTE

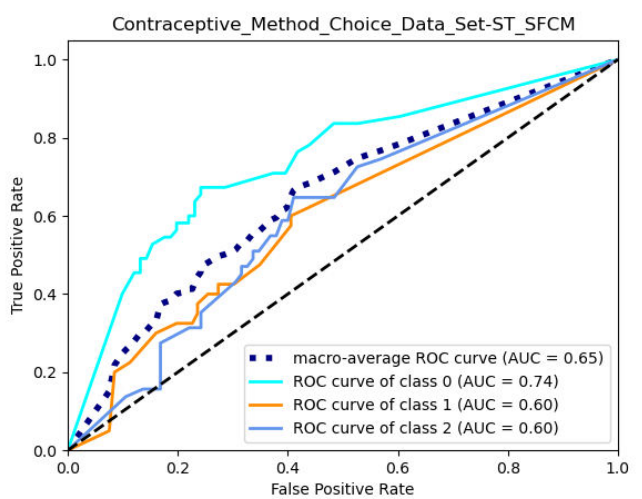

(d)ST_SFCM

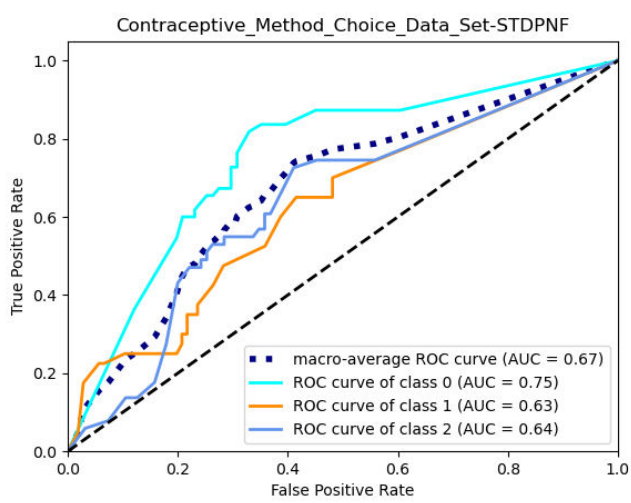

(f)STDPNF

Fig. 5 ROC of Contraceptive_Method_Choice data set

\subsection{Experiment 2: comparisons between the proposed noise filter and existing ones}

In order to prove the effectiveness of GAMNF, we selected 6 representative noise filters ENN (2017), RNN (1967), ALLKNN (2019), MENN (2000), CEWS (2020), PNF (2019) for comparison. To compare their abilities of solving the problem of mislabeling, STDP algorithm is chosen to combine the 6 noise filters (STDP_ENN, STDP_RNN, STDP_ALLKNN，STDP_MENN，STDP_CEWS，STDP_PNF). 
Table 6 shows the comparison algorithm and related parameters.

Experiment 2 uses the kappa coefficient to judge the quality of the noise filter, which can not only check the consistency and measure the classification accuracy, but also judge the ability of unbalanced classification (W. Pei et al. 2020). The calculation method of kappa coefficient is shown in Eq. (9) where $p_{o}$ is classification accuracy. The calculation method of $p_{e}$ is shown in Eq. (10), where the total number of samples is $n$, the number of real samples in each class is $a_{1}, a_{2}, \ldots, a_{c}$, and the predicted number of samples in each class is $b_{1}, b_{2}, \ldots, b_{c}$.

$$
\begin{aligned}
& \mathrm{k} \text { a p p=a } \frac{p_{o}-p_{e}}{1-p_{e}} \\
& \mathrm{p}_{e}=\frac{a_{1} \times b_{1}+a_{2} \times b_{2}+\ldots+a_{c} \times b_{c}}{n \times n}
\end{aligned}
$$

Table 7 and Table 8 shows the experimental results of GAMNF in STDP and other comparison algorithms. The base classifiers in Table 7 and Table 8 are respectively selected as KNN and CART.

From Table 7 and Table 8 , when the base classifier is KNN, 11 out of 16 data sets' kappa coefficient of our algorithm are better than the comparison algorithms. When the base classifier is CART, 7 out of 16 data sets' kappa coefficient of our algorithm are better than the comparison algorithm. In summary, the overall accuracy of STDP_GAMLNF is higher than the comparison algorithm, and the effect is better when the base classifier is KNN. However, the performances of the two base classifiers on the MM and TTTE data sets perform not well. In particular, all the noise filters on the TTTE data set lead to the accuracy reduction, because the spatial structure revealed by DPC has been sufficient to support the training of the self-training algorithm. Furthermore, the noise filter will interfere the relabel samples, resulting in the deletion of too many unlabeled samples, which will corrupt the complete spatial structure. While STDP_GAMLNF can effectively detect mislabeled samples by multi-local mean vectors, and uses harmonic average distance to focus on local mean vectors close to the relabeled samples in each class, so as to achieve better detection ability.

Table 6 Comparison algorithms and parameters in experiment 2.

\begin{tabular}{cll}
\hline Abbreviation & \multicolumn{1}{c}{ Algorithms } & \multicolumn{1}{c}{ Parameters } \\
\hline STDP & Self-training with DPC & $P_{a}=2$. \\
STDP_ENN & STDP combining ENN & $P_{a}=2, k=3$. \\
STDP_RENN & STDP combining RENN & $P_{a}=2, k=3$. \\
STDP_ALLKNN & STDP combining ALLKNN & $P_{a}=2, k=3$. \\
STDP_MENN & STDP combining MENN & $P_{a}=2, k=3$. \\
STDPNF & STDP combining PNF & $P_{a}=2$. \\
STDP_CEWS & STDP combining CEWS & $P_{a}=2, \theta=0.1$. \\
STDP_GAMLNF & Our algorithm & $P_{a}=2, r=k \times n(n$ is the number of classes $)$ \\
\hline
\end{tabular}


Self-training algorithm based on density peaks combining globally adaptive multi-local noise filter

Table 7 Experimental results of kappa with KNN as base classifier.

\begin{tabular}{ccccccccc}
\hline \multirow{2}{*}{ Data sets } & STDP & $\begin{array}{c}\text { STDP_ } \\
\text { ENN }\end{array}$ & $\begin{array}{c}\text { STDP_ } \\
\text { RENN }\end{array}$ & $\begin{array}{c}\text { STDP_AL } \\
\text { LKNN }\end{array}$ & $\begin{array}{c}\text { STDP_ } \\
\text { MENN }\end{array}$ & STDP_PNF & STDP_CEWS & Ours \\
\hline ACA & 0.3632 & 0.3466 & 0.3466 & 0.3467 & 0.3466 & 0.3645 & 0.3685 & $\mathbf{0 . 3 7 6 1}$ \\
BIO & 0.4885 & 0.4806 & 0.4806 & 0.4823 & 0.4806 & 0.4996 & 0.4882 & $\mathbf{0 . 5 0 4}$ \\
BTSC & 0.2700 & 0.2605 & 0.2605 & 0.2605 & 0.2605 & 0.27 & 0.2641 & $\mathbf{0 . 2 8 2 9}$ \\
AVI & 0.6246 & 0.6244 & 0.6248 & 0.6363 & 0.6244 & 0.6301 & 0.6234 & $\mathbf{0 . 6 6 1 7}$ \\
CMCDS & 0.2598 & 0.24 & 0.24 & 0.238 & 0.2357 & 0.2535 & 0.2375 & $\mathbf{0 . 2 6 3 8}$ \\
GAU & 0.7945 & $\mathbf{0 . 7 9 9 3}$ & $\mathbf{0 . 7 9 9 3}$ & 0.7702 & $\mathbf{0 . 7 9 9 3}$ & 0.7945 & 0.7747 & 0.7886 \\
GER & 0.2754 & 0.2699 & 0.2699 & 0.2313 & 0.2699 & 0.2664 & 0.2655 & $\mathbf{0 . 2 7 7 2}$ \\
IS & 0.8738 & 0.8753 & 0.8753 & 0.8819 & 0.8758 & 0.8738 & 0.8723 & $\mathbf{0 . 8 9 1}$ \\
ILPD & 0.2679 & 0.2312 & 0.2312 & 0.2795 & 0.2312 & 0.2665 & $\mathbf{0 . 2 8 5 5}$ & 0.2632 \\
MM & 0.5771 & 0.5834 & 0.5834 & 0.5859 & $\mathbf{0 . 5 9 5 4}$ & 0.5732 & 0.5928 & 0.5711 \\
SEG & 0.8546 & 0.8586 & 0.8586 & 0.8667 & 0.8586 & 0.8596 & 0.8606 & $\mathbf{0 . 8 8 3 4}$ \\
SPA & 0.4455 & 0.4413 & 0.4418 & 0.4288 & 0.4417 & 0.4381 & 0.4409 & $\mathbf{0 . 4 7 2 1}$ \\
TTTE & 0.1919 & $\mathbf{0 . 2 5 7 3}$ & $\mathbf{0 . 2 5 7 3}$ & 0.2414 & 0.2158 & 0.223 & 0.1894 & 0.2511 \\
WP & 0.7362 & $\mathbf{0 . 7 4 8}$ & $\mathbf{0 . 7 4 8}$ & 0.747 & 0.746 & 0.74 & 0.7363 & 0.7316 \\
WAV & 0.7554 & 0.7553 & 0.7553 & 0.7533 & 0.755 & 0.7578 & 0.7458 & $\mathbf{0 . 7 6 9 2}$ \\
WIL & 0.9611 & 0.9618 & 0.9618 & 0.9625 & 0.9618 & 0.9618 & 0.9625 & $\mathbf{0 . 9 7 1 8}$ \\
\hline
\end{tabular}

Table 8 Experimental results of kappa with CART as base classifier.

\begin{tabular}{ccccccccc}
\hline Data sets & STDP & $\begin{array}{c}\text { STDP_ } \\
\text { ENN }\end{array}$ & $\begin{array}{c}\text { STDP_ } \\
\text { RENN }\end{array}$ & $\begin{array}{c}\text { STDP_AL } \\
\text { LKNN }\end{array}$ & $\begin{array}{c}\text { STDP_- } \\
\text { MENN }\end{array}$ & STDP_PNF & STDP_CEWS & Ours \\
\hline ACA & 0.6698 & 0.6602 & 0.6836 & 0.6655 & 0.66 & 0.6698 & 0.6698 & $\mathbf{0 . 6 7 2 7}$ \\
BIO & 0.6197 & 0.6408 & 0.6312 & 0.6096 & 0.6408 & $\mathbf{0 . 6 4 1 2}$ & 0.6015 & 0.6338 \\
BTSC & 0.2895 & 0.2813 & 0.2813 & 0.2809 & 0.2813 & 0.2622 & 0.2755 & $\mathbf{0 . 2 9 3 6}$ \\
AVI & 0.8739 & 0.8425 & 0.8327 & 0.8319 & 0.8425 & 0.8695 & $\mathbf{0 . 8 7 7 2}$ & 0.8683 \\
CMCDS & 0.3009 & 0.3179 & 0.316 & 0.3017 & $\mathbf{0 . 3 2 3 8}$ & 0.2778 & 0.3078 & 0.3125 \\
GAU & 0.4367 & 0.5616 & 0.5451 & 0.5487 & 0.5604 & 0.478 & 0.4769 & $\mathbf{0 . 5 6 6 5}$ \\
GER & 0.3307 & 0.3556 & 0.3489 & 0.3439 & 0.3555 & $\mathbf{0 . 3 5 9 7}$ & 0.3594 & 0.3367 \\
IS & 0.922 & 0.9250 & 0.926 & $\mathbf{0 . 9 4 1 2}$ & 0.9235 & 0.9270 & 0.928 & 0.9316 \\
ILPD & 0.2278 & 0.2857 & 0.2429 & 0.2385 & 0.2974 & 0.2664 & 0.2599 & $\mathbf{0 . 2 9 7 7}$ \\
MM & 0.6675 & $\mathbf{0 . 6 9 7 1}$ & 0.6751 & 0.6679 & 0.6522 & 0.6703 & 0.672 & 0.6337 \\
SEG & 0.9219 & 0.9123 & 0.9245 & 0.9219 & 0.9123 & 0.9229 & 0.9235 & $\mathbf{0 . 9 3 2 1}$ \\
SPA & 0.7684 & 0.7872 & 0.7858 & $\mathbf{0 . 8 0 0 2}$ & 0.7955 & 0.7764 & 0.7648 & 0.7792 \\
TTTE & $\mathbf{0 . 3 5 7}$ & 0.3421 & 0.2522 & 0.3396 & 0.3303 & 0.3461 & 0.3546 & 0.3474 \\
WP & 0.7786 & 0.764 & 0.769 & 0.7775 & 0.7576 & 0.7742 & 0.779 & $\mathbf{0 . 7 9 8 5}$ \\
WAV & 0.6691 & 0.7056 & 0.7177 & 0.7181 & 0.7056 & 0.675 & 0.6712 & 0.6989 \\
WIL & 0.8983 & 0.9391 & 0.9398 & $\mathbf{0 . 9 4 1 1}$ & 0.9391 & 0.9304 & 0.9003 & $\mathbf{0 . 9 4 1 1}$ \\
\hline
\end{tabular}

\subsection{Experiment 3: sensitivity of noise filters to the neighborhood $\boldsymbol{k}$ value}

In GAMLNF $r$ depends on $k$, which should be specially concentrated on. Therefore, GAMLNF only need to consider whether it is sensitive to $k$ or not. The compared algorithm selects noise filters with $k$ values, such as ENN, RNN, ALLKNN, and MENN. In order to better compare the sensitivity of 
each noise filter to the value of $k, k$ is tested and from 1 to 10 , the average accuracy of $k$ is used to find the variance. The smaller the variance is, the less sensitive the noise filter is to $k$.

The experimental results are shown in Figure 6. It is clear that the variance of GAMLNF is much lower than other noise filters, and it can achieve an adaptive global search for the appropriate $k_{i}$ for each class. Although GAMLNF is not as stable as ALLKNN on the ILPD data set, it has a better classification effect. Single pseudo-average vector is obtained for $k_{i}$ local average vector in each class, and the true class of the relabeled samples by pseudo-average vectors is obtained.

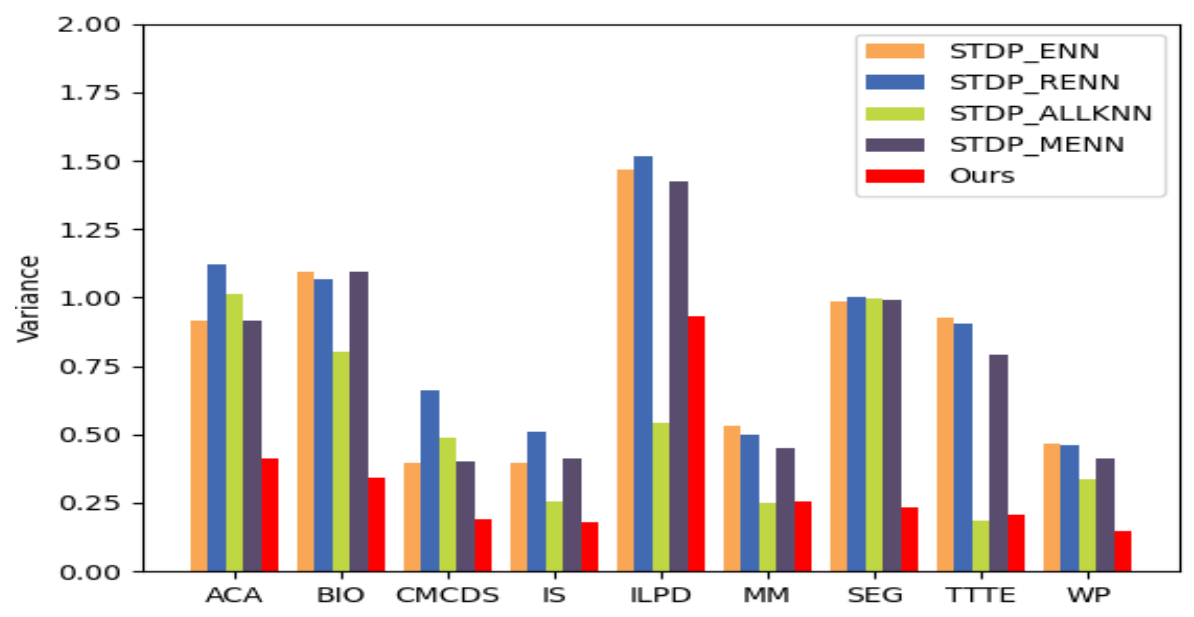

Fig. 6 Variance results of different $K$ values

\subsection{Experiment 4: the impact of ratio of labeled samples}

The experimental parameters are consistent with Table 6. Simultaneously, STDP_MENN, STDP_PNF and STDP_GAMLNF perform better than other algorithms in Experiment 2, so they are used as compared algorithms. Figure 7 shows that the ratio of $\boldsymbol{L}(10 \%$ 90\%) affects the average accuracy in different data sets.

As shown in Figure 7, GAMLNF performs better than the comparison algorithm on ACA, BIO, IS, ILPD, SEG, and TTTE data sets. GAMLNF calculates the most suitable local average vector for each class globally, which can achieve the effect of improving the classification accuracy through multiple average center. But on IS, ILPD, and TTTE data sets, when the ratio of $\boldsymbol{L}$ is $10 \%$, the classification effect is significantly lower than the compared algorithms. GAMLNF need to find local average vector in each class, and if the ratio of $\boldsymbol{L}$ is too small, GANN can not find enough local neighbors in each class, and the global neighbors belong to a certain class. It is especially important to note that all algorithms can increase the accuracy of the algorithm when the labeled samples ratio increase, especially when the ratio of $\boldsymbol{L}$ is from $10 \% \sim 50 \%$. 


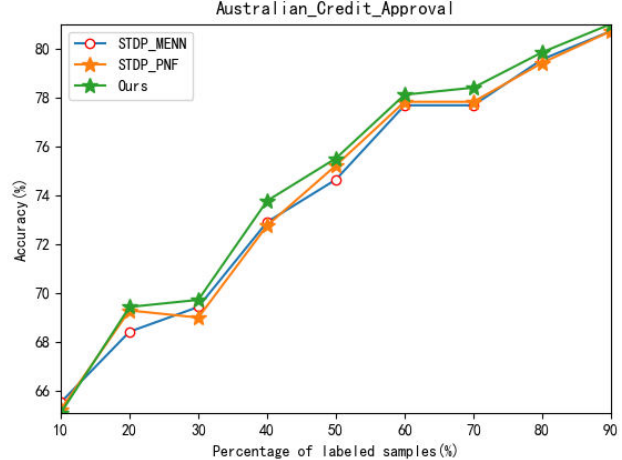

(a)ACA data set

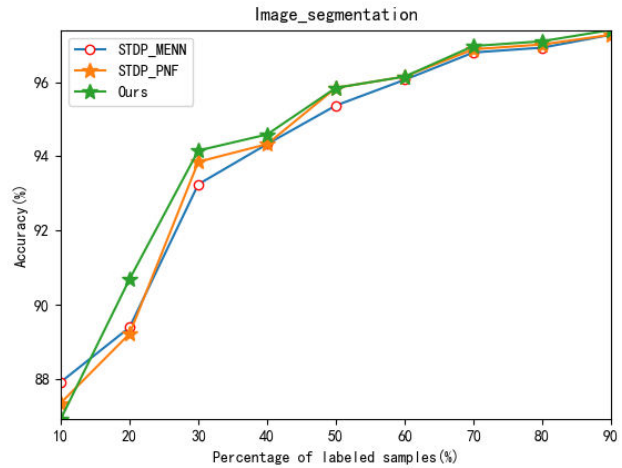

(c)IS data set

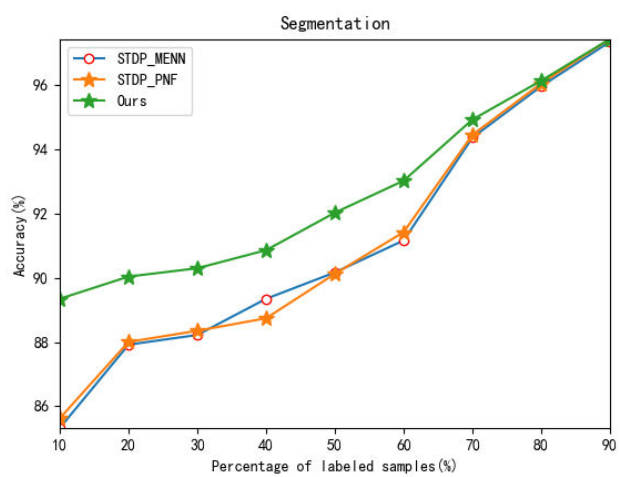

(e)SEG data set

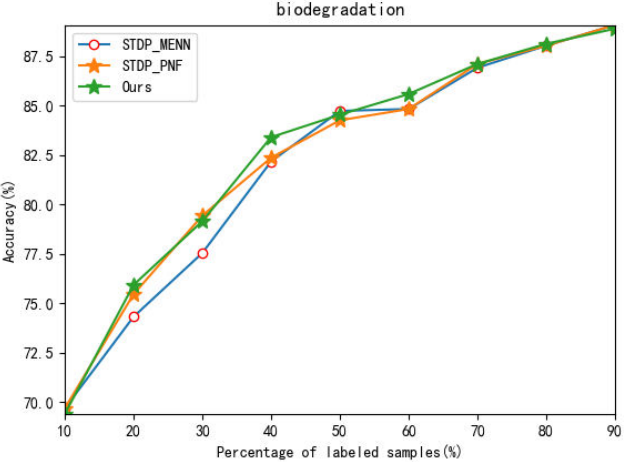

(b)BIO data set

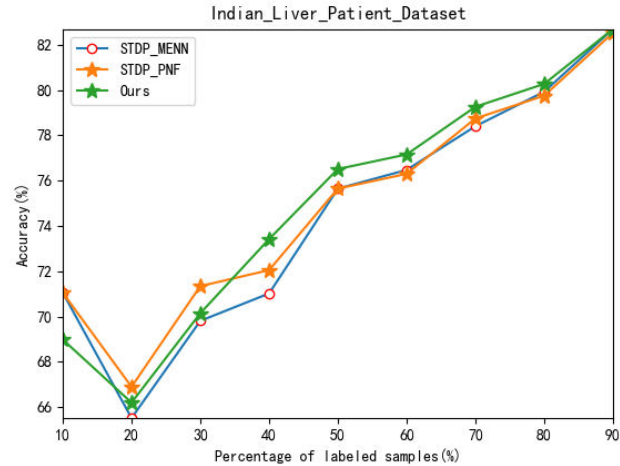

(d)ILPD data set

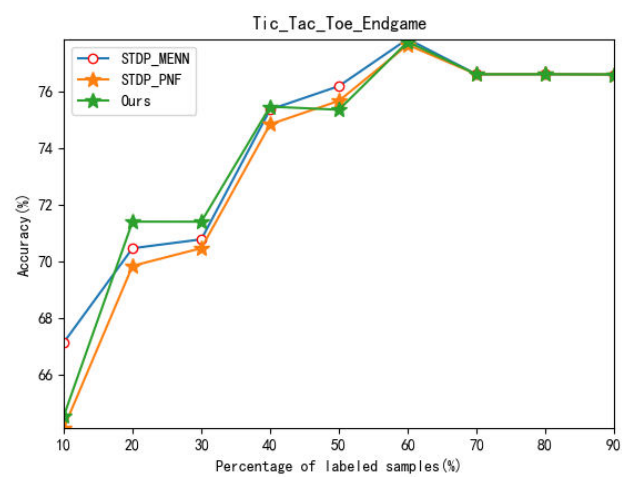

(f)TTTE data set

Fig. 7 ACC of different algorithms with respect to different percentages of L on 6 data sets

\section{Conclusions and future study}

In this paper, we firstly explain in detail how we use GAMLNF to detect mislabeled samples. Then, we introduce how to repair the spatial structure damaged by the noise filter. In the end, we propose our STDP-GAMLNF algorithm framework. Specifically, the spatial structure of the data sets is revealed by DPC, and then the spatial structure is used to help self-training to label unlabeled samples. Meanwhile, GAMLNF is used to detect whether the relabeled sample is mislabeled sample or not,after each epoch of 
labeling. SSR technology can reconstruct the corrupted space structure. Besides, all the experiments clearly show that (a) Compared with mainstream methods, the proposed algorithm can effectively improve the average accuracy and AUC value. (b) GAMLNF's ability to detect mislabeled samples is better than other comparison algorithms. (c) The presented algorithm is not sensitive to the neighbor parameter $k$ value and can be adaptive to find the appropriate number of neighbors of each class. (d) The average accuracy of the algorithm will be improved when the ratio of $\boldsymbol{L}$ increases, and it can be better than other comparison algorithms in most cases.

In future work, we will consider how to improve the noise filter to promote the detection of mislabeled samples, when there exist extremely few labeled samples. We find that the increasing number of labeled samples can significantly improve the accuracy of the algorithm. Therefore, we also consider how to relabel the incorrectly labeled samples through the updated spatial structure for the sake of making the labeled samples not be simply deleted.

\section{Compliance with ethical standards}

Ethical approval This article does not contain any studies with human participants or animals performed by any of the authors.

Funding details This work is supported by the scientific and technological innovation project of double-city economic circle construction in Chengdu-Chongqing area (No.KJCX2020024), Chongqing University Innovation Research Group funding (No.CXQT20015)

Conflict of interest The authors declare that they have no conflicts of interest.

Informed consent Informed consent was obtained from all individual participants included in the study. Authorship contributions

Shuaijun Li: Conceptualization, Methodology, Validation, Formal analysis, Data Curation, WritingOriginal Draft.

Jia Lu: Supervision, Writing - Review \& Editing.

\section{References}

Li Y, Pan Q, Wang S, et al (2019) disentangled variational auto-encoder for semi-supervised learning. information sciences 482(12): 73-85.

Yuan C, Chen X, Yu P, et al (2020) semi-supervised stacked autoencoder-based deep hierarchical semantic feature for real-time fingerprint liveness detection. Journal of Real-Time Image Processing 17(1): 55-71.

Tran VC, Nguyen NT, Fujita H, Hoang DT, Hwang D (2017) a combination of active learning and self-learning for named entity recognition on twitter using conditional random fields. Knowledge-Based Systems 132: 179-187

Liu Y, Shi H, Du H et al (2021) boosting semi-supervised face recognition with noise robustness. IEEE Transactions on Circuits and Systems for Video Technology 99: 10-18.

Zhang M, Yang Z, Liu C and Fang L (2020) traditional Chinese medicine knowledge service based on semi-supervised BERT-BiLSTM-CRF model. 2020 International Conference on Service Science pp:64-69.

Li J, Zhu Q (2019) semi-supervised self-training method based on an optimum-path forest. IEEE Access 7(3): 36388-36399.

Pande N, Awate S. P. (2021) generative deep-neural-network mixture modeling with semi-supervised 
MinMax+EM learning. 25th International Conference on Pattern Recognition pp:5666-5673.

Slivka J, Sladic G, Milosavljevic B, et al (2017) a tool for flexible experimenting with co-training based semi-supervised algorithms, Knowledge-Based Systems 121(1): 4-12.

Anis A, Gamal A E, Avestimehr A S, et al (2019) a sampling theory perspective of graph-based semi-supervised learning. IEEE Transactions on Information Theory 65(4): 2322-2342.

Gan H, Sang N, Huang R, Tong X, Dan Z (2013) using clustering analysis to improve semi-supervised classification. Neurocomputing 101 (3): 290-298.

Wu D, Shang M, Wang G, et al (2018) a self-training semi-supervised classification algorithm based on density peaks of data and differential evolution. IEEE 15th International Conference on Networking, Sensing and Control (ICNSC). IEEE: 1-6.

Li J, Zhu Q, Wu Q (2019) a self-training method based on density peaks and an extended parameter-free local noise filter for $\mathrm{k}$ nearest neighbor. Knowledge-Based Systems 184(3): 104-113.

Wei D, Y Yang, Qiu H (2020) improving self-training with density peaks of data and cut edge weight statistic. Soft Computing 24: 15595-15610.

Wei Z, Wang H, Zhao R (2013) semi-supervised multi-label image classification based on nearest neighbor editing. Neurocomputing 119: 462-468.

Triguero I, Sáez J.A, Luengo J, García S, Herrera F (2014) on the characterization of noise filters for self-training semi-supervised in nearest neighbor classification. Neurocomputing 132: 30-41.

Mateos-Garc1'a D, Garc1'a-Gutie'rrez J, Riquelme-Santos JC (2016) an evolutionary voting for k nearest neighbors. Expert Syst Appl 43:9-14.

Mitani Y, Hamamoto Y (2006) A local mean-based nonparametric classifier. Pattern Recognit Lett 27(10):1151-1159.

Gou J, Zhan Y, Rao Y, Shen X, Wang X, He W (2014) improved pseudo nearest neighbor classifification. Knowl Based Syst 70(C): 361-375.

Pan Z , Pan Y, Wang Y, et al (2020) a new globally adaptive k -nearest neighbor classifier based on local mean optimization. Soft Computing: 1-15.

Pan Z, Wang Y, Ku W (2017a) a new general nearest neighbor classifification based on the mutual neighborhood information. Knowl Based Syst 121:142-152.

Pan Z, Wang Y, Ku W (2017b) a new k-harmonic nearest neighbor classififier based on the multi-local means. Expert Syst Appl 67:115-125.

Li M, Zhou Z.H (2005) SETRED: self-training with editing. Pacific-asia conference on advances. knowledge Discovery \& Data Mining pp: 611-621.

Wei Z, Wang H, Zhao R (2013) semi-supervised multi-label image classification based on nearest neighbor editing. Neurocomputing 119: 462-468.

Asuncion A, Newman D (2007) UCI machine learning repository. Available : http://archive.ics.uci.edu/ml/datasets.php.

Wu D, Shang M.S, Luo X, Xu J, Yan H.Y, Deng W.H, et al (2017) self training semi-supervised classification based on density peaks of data. Neurocomputing 275: 180-191.

Tomek I (1967) An experiment with the edited nearest-neighbor rule. IEEE Trans. Syst. Man Cybern. 6(6): 448-452.

Hattori K, Takahashi M (2000) a new edited k-nearest neighbor rule in the pattern classification problem. Pattern Recognit 33(3): 521-528. 
Self-training algorithm based on density peaks combining globally adaptive multi-local noise filter

Pei W, Xue B, Shang L and Zhang M (2020) a threshold-free classification mechanism in genetic programming for high-dimensional unbalanced classification. IEEE Congress on Evolutionary Computation (CEC) pp: 1-8. 